\title{
30 ans d'articles dans Mappemonde. Une exploration par les mots-clés et les résumés
}

30 years of articles in Mappemonde. An exploration of keywords and abstracts Treinta años de artículos en Mappemonde. Un análisis a través de las palabras clave y los resúmenes.

Grégoire Le Campion, Olivier Pissoat, Laurent Jégou et Matthieu Noucher

\section{OpenEdition}

\section{Journals}

Édition électronique

URL : http://journals.openedition.org/mappemonde/2494

DOI : 10.4000/mappemonde.2494

ISSN : 1769-7298

Éditeur

UMR ESPACE

\section{Référence électronique}

Grégoire Le Campion, Olivier Pissoat, Laurent Jégou et Matthieu Noucher, « 30 ans d'articles dans

Mappemonde. Une exploration par les mots-clés et les résumés », Mappemonde [En ligne], 127 | 2019, mis en ligne le 01 juillet 2019, consulté le 29 mars 2020. URL : http://journals.openedition.org/

mappemonde/2494; DOI : https://doi.org/10.4000/mappemonde.2494

Ce document a été généré automatiquement le 29 mars 2020.

\section{cc) (1) ()}

La revue Mappemonde est mise à disposition selon les termes de la Licence Creative Commons Attribution - Pas d'Utilisation Commerciale - Partage dans les Mêmes Conditions 4.0 International. 


\title{
30 ans d'articles dans Mappemonde. Une exploration par les mots-clés et les résumés
}

\author{
30 years of articles in Mappemonde. An exploration of keywords and abstracts \\ Treinta años de artículos en Mappemonde. Un análisis a través de las palabras \\ clave y los resúmenes.
}

Grégoire Le Campion, Olivier Pissoat, Laurent Jégou et Matthieu Noucher

\section{Introduction}

1 En s'intéressant aux représentations spatiales sous toutes leurs formes (cartes, croquis et cartogrammes, modèles graphiques, images traitées, représentations 3D, animées, dynamiques, etc.), Mappemonde est progressivement devenue une revue française de référence sur l'image géographique. Sans se restreindre à une discipline, ses articles publiés tous les trimestres dans les domaines les plus divers de l'écologie, de l'archéologie, de la sociologie, de l'art, de l'urbanisme, de l'architecture, etc. permettent d'appréhender à la fois la place de l'iconographie comme support de la recherche, mais aussi comme objet de recherche. Il est bien difficile pour le lecteur d'en avoir une vision synthétique et dynamique tant le contenu de la revue est foisonnant. Revenir sur 30 ans de publication d'articles dans Mappemonde c'est, en effet, se confronter à un corpus conséquent de 124 numéros rassemblant 964 articles, publiés sur trois supports dématérialisés différents : une page web rassemblant les archives des numéros imprimés de 1986 à $2003^{1}$, un site offrant un accès aux articles de 2004 à $2015^{2}$ et le site actif entre 2016 et $2019^{3}$.

2 Pour tenter d'explorer ce contenu, nous proposons, dans un premier temps, une approche automatisée pour l'extraction puis l'étude statistique des mots-clés des articles. Leur analyse fournit alors des éléments intéressants pour dresser un rapide panorama temporel des objets et champs thématiques couverts par la revue, mais elle 
permet également de révéler les limites d'une telle approche. En effet, si les mots-clés sont obligatoires pour toute publication dans la revue, ces derniers ne sont pas normalisés, i.e. les auteurs n'ont pas à les choisir dans une nomenclature établie a priori. Les analyses a posteriori limitent ainsi le potentiel heuristique de ce corpus. Dans un deuxième temps, nous avons par conséquent procédé à une analyse complémentaire, cette fois sur les résumés, pour tenter d'affiner notre appréhension à partir de cet ensemble de textes.

\section{Exploration des mots-clés}

Pour un article scientifique, les mots-clés doivent décrire précisément le contenu de la contribution. Il s'agit le plus souvent des mots essentiels tirés de l'article, mais ils peuvent prendre la forme d'expressions (groupes nominaux de 2 ou 3 mots). Ils indiquent, par exemple, l'objet d'étude, les concepts fondamentaux qui sont mobilisés, les méthodes utilisées, les terrains concernés (Fovet-Rabot, 2015). Leur analyse peut donc aider à déceler des tendances et, in fine, à porter un regard réflexif sur la ligne éditoriale d'une revue.

Dans le cas qui nous intéresse, les 964 articles publiés par Mappemonde depuis sa création ${ }^{4}$ permettent de constituer un corpus de 4635 occurrences (soit 4,7 mots-clés par article). Un premier comptage sans nettoyage ou regroupement des données dénombre 2445 mots-clés différents et révèle les huit mots-clés les plus cités (plus de 30 fois). Cependant, bien qu'il s'agisse d'une distribution assez classique en bibliométrie, la distribution asymétrique des fréquences de ces occurrences (figure 1) est telle qu'elle attire l'attention sur la quantité inattendue de mots-clés cités une seule fois (près de 40\%). Combinée aux effets de la loi de Zipf, donc, c'est sans doute l'absence de normalisation qui conduit à un tel résultat : les auteurs ne devant pas choisir leurs mots-clés dans un thésaurus, force est de constater un éparpillement des saisies (pourtant parfois très proches lorsque, par exemple, des termes sont utilisés au singulier et au pluriel).

Figure 1. Distribution des mots-clés

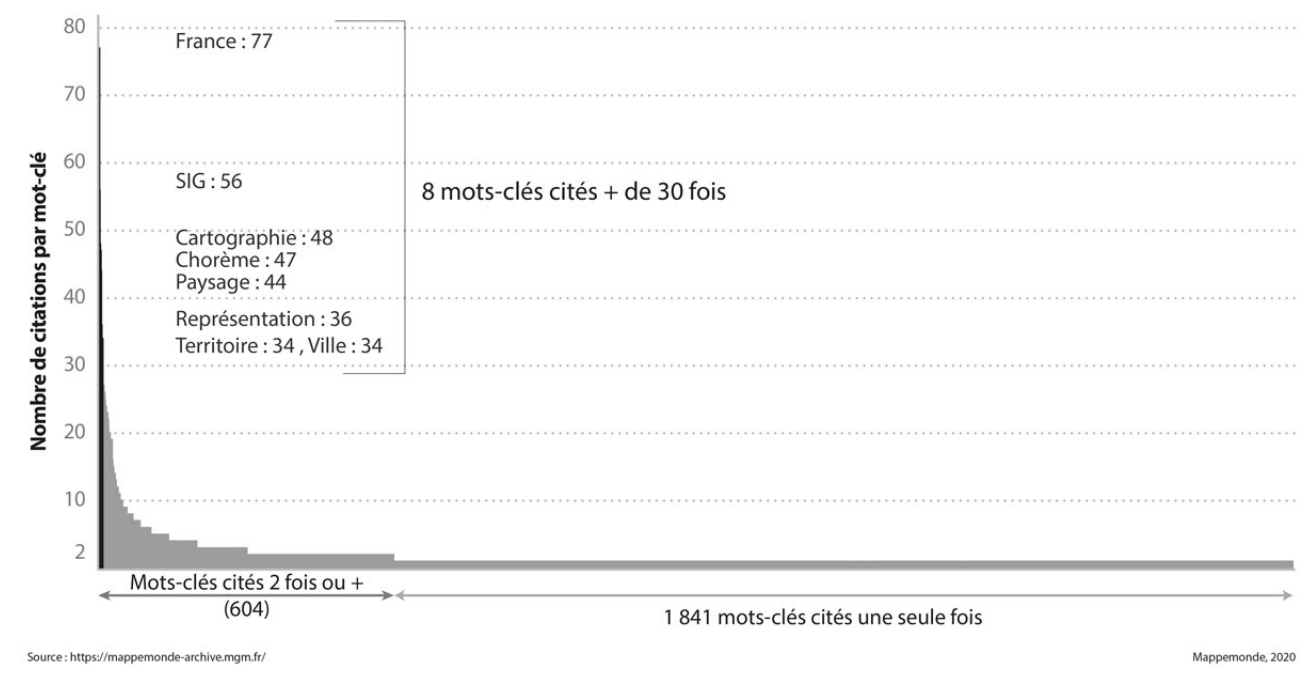


Un travail manuel de catégorisation a donc semblé nécessaire pour aller plus loin dans l'étude. Pour gagner du temps et nous focaliser sur le cœur de l'analyse, nous avons choisi de ne traiter que les mots-clés cités au moins deux fois (604 mots-clés, cités 2794 fois, soit $25 \%$ du corpus, mais $60 \%$ des occurrences). Un regroupement en douze catégories a alors été opéré. Certaines associations sont apparues comme évidentes (pédagogie ; didactique; géographie scolaire; didactique de la géographie); d'autres relèvent de choix qui ont demandé plus de réflexion (modélisation; chorème; modèle graphique). Le travail en équipe s'est révélé ici particulièrement utile pour confronter nos propositions et arriver à des consensus sur les catégories et les regroupements. La figure 2 présente les effectifs de cette catégorisation en dix classes.

Figure 2. Classification des mots-clés cités au moins deux fois

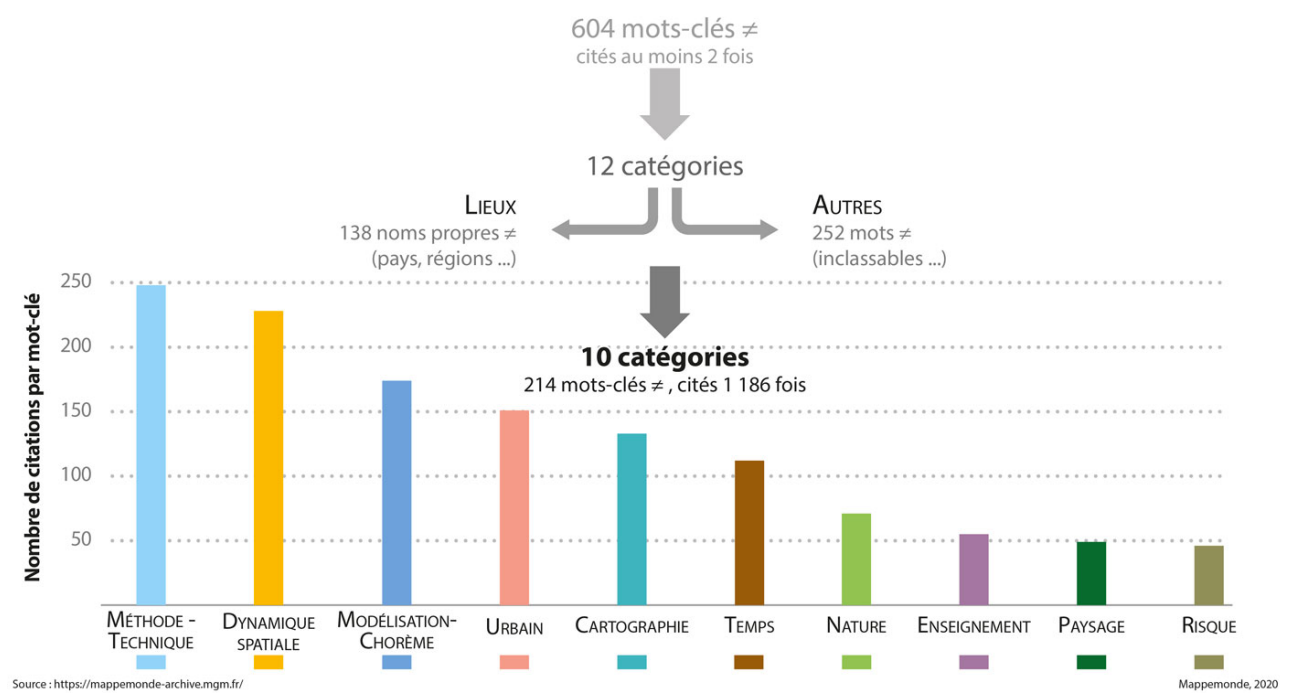

Même après les regroupements effectués, des invariants demeurent par rapport aux thèmes suggérés par les mots-clés recensés plus de 30 fois : la place accordée à la modélisation chorématique, à l'urbain ou au paysage notamment. En revanche, d'autres thématiques apparaissent, autour de la notion de temps, de la nature ou des risques par exemple. Cependant, deux catégories se détachent quelque peu. La première concerne les aspects méthodologiques et techniques. Elle constitue la classe la plus nombreuse, car elle regroupe tout ce qui a trait à la télédétection et au travail sur l'image, mais aussi, surtout tous les éléments relatifs à la géomatique et aux systèmes d'information géographique, qui sont fréquents, pour preuve le score obtenu lors du premier traitement par le sigle devenu acronyme, SIG. La deuxième catégorie s'attache aux dynamiques spatiales : y sont rassemblés les mots-clés décrivant les processus à l'œuvre dans l'évolution et l'organisation des espaces et territoires (figure 3). 
Figure 3. Les mots-clés par catégorie

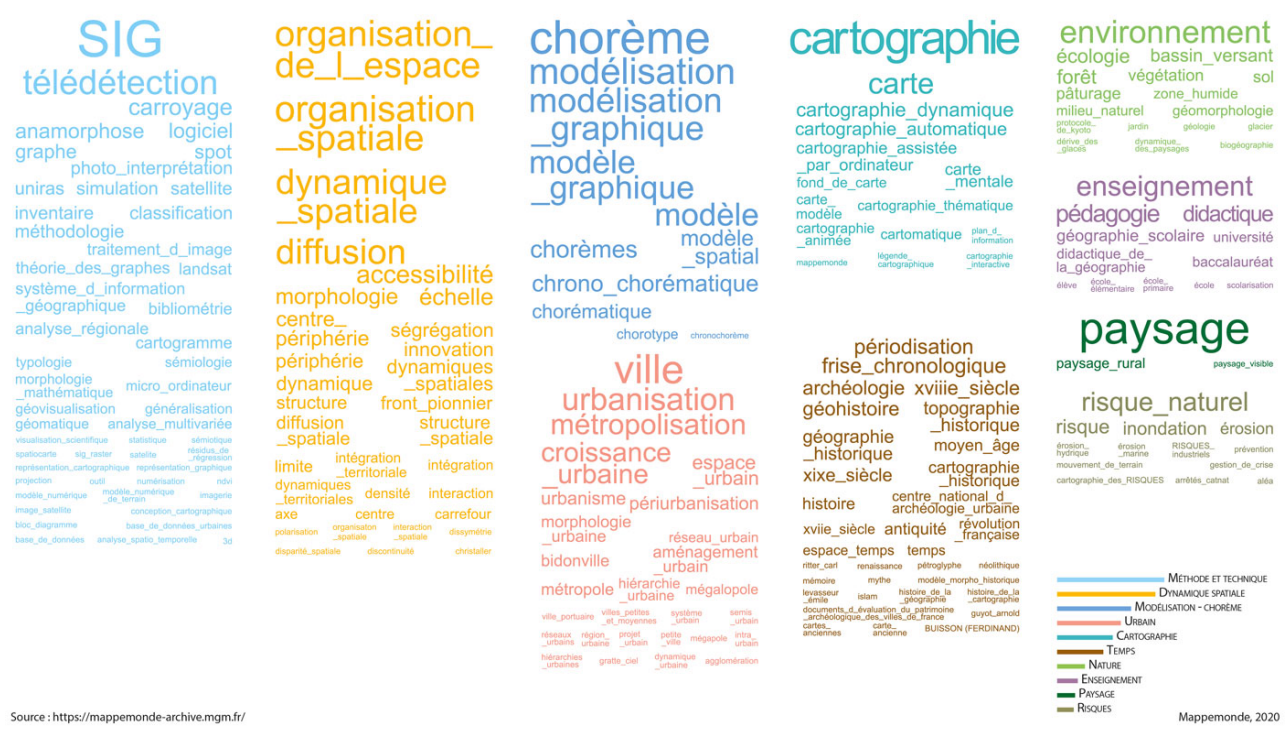

7 Nous avons fait le choix d'isoler les mots-clés centrés sur la cartographie stricto sensu. Qu'elle soit thématique, statistique, de synthèse, automatique, assistée par ordinateur, animée ou bien encore dynamique, elle fait partie de l'ADN de la revue comme en atteste l'éditorial du premier numéro signé par Roger Brunet. Intitulé « Mappemonde, le Monde des Cartes ", il annonce d'emblée la couleur au $2^{\mathrm{e}}$ paragraphe : «La demande est claire : parmi les images, des cartes.» (Brunet, 1986:1) et utilise 15 fois le mot « carte » dans cet article d'une page.

8 L'analyse de l'évolution de l'usage de ces catégories met en évidence certaines continuités au cours des 30 années étudiées, mais aussi ce qu'on pourrait appeler des «épisodes scientifiques » (figure 4). En effet, les mots-clés renvoyant à la "méthode » et aux questions de "dynamique spatiale » sont très utilisés quasiment tout au long de la période. Il en est de même, dans une moindre mesure, de l'entrée par l'urbain qui occupe une place importante et assez continue. Elle rejoint en 2010 l'une des figures emblématiques de l'identité de Mappemonde : les chorèmes et modèles. Du reste, la première figure publiée dans la revue (1986/1: https://www.mgm.fr/PUB/ Mappemonde/M186/p2-3.pdf) est un modèle graphique (une composition de chorèmes) des structures et dynamique de l'espace en Espagne et en France. Il est proposé par Lise Forton pour tester la grille utilisée par Roger Brunet (1974) au-delà de l'Hexagone. C'est le début d'une longue série qui se poursuit aujourd'hui et dont on peut relever trois moments forts : en 1990, Jean-Pierre Deffontaines, Jean-Paul Cheylan et Sylvie Lardon coordonnent un dossier thématique (4/1990: https://www.mgm.fr/PUB/ Mappemonde/Mappe490R.html) intitulé « Gestion de l'espace rural, des pratiques aux modèles "; entre 1994 et 1996, les contributions sur les chorèmes représentent 12 à 15 articles par an, sans pour autant qu'un dossier thématique leur soit consacré, ce qui témoigne alors de la vivacité de l'approche; enfin, en 2010 se produit un épisode particulier, lorsque le dossier du $n^{\circ} 100$ dédié à la "chrono-chorématique urbaine " (4/2010: https://mappemonde-archive.mgm.fr/dos_chrono.html), coordonné par Xavier Rodier, Christian Grataloup et Corinne Guilloteau, remet à nouveau au centre de la ligne éditoriale la discussion sur « ces modèles généraux de la production de l'espace géographique ", pour reprendre l'expression de Roger Brunet qui conclut ce dossier (Brunet, 2010) . 
Figure 4. Évolution des dix catégories de mots-clés (1986-2017)
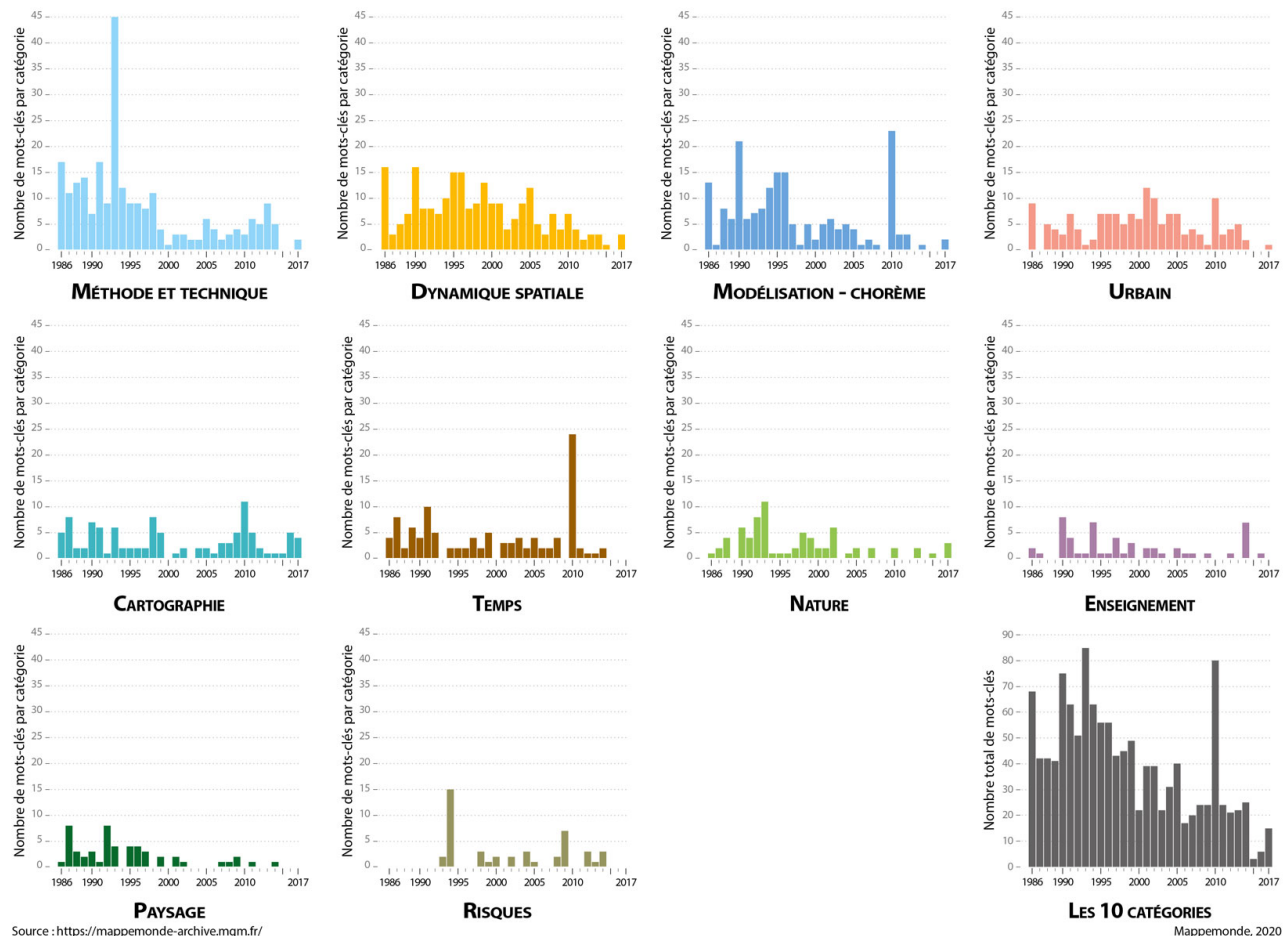

Dans les autres catégories se distinguent pareillement des sortes de «vagues thématiques ». Ainsi, l'enseignement de la géographie est un thème récurrent qui fait l'objet d'une attention redoublée à différents moments, avec des dossiers thématiques comme «Enseigner la carte» (3/1990: https://www.mgm.fr/PUB/ Mappemonde/Mappe390.html) ou plus récemment «La géographie scolaire. De l'école élémentaire aux filières universitaires professionnalisantes » (113/2014: https:// mappemonde-archive.mgm.fr/num41/index.html).

La thématique du paysage, présente dès les débuts de la revue, semble moins traitée dans les articles avec, durant les années 2000 , des contributions très ponctuelles. Ce constat est à nuancer avec la présence de cette thématique dans les Prix de Thèse ou encore dans la rubrique "Images », deux éléments importants de Mappemonde qui ne sont pas analysés ici.

11 La problématique des risques apparait pour la première fois en 1993 avec un article de Asté, Dumolard, Léone et Jin sur l'utilisation d'un système d'information géographique pour cartographier différents scénarios de glissement de terrain sur le massif de l'Oisans (Isère) $)^{5}$. Un dossier y est consacré un an plus tard (1994/4: https:// www.mgm.fr/PUB/Mappemonde/Mappe494.html), ce qui explique le pic observé sur l'histogramme. Depuis, cette thématique revient régulièrement.

Plus globalement, cumuler les 10 catégories livre une information tout aussi instructive. À partir des années 2000, ces thématiques semblent moins présentes dans les publications de Mappemonde. Cette baisse relative signale l'ouverture grandissante de la revue à de multiples et nouveaux sujets. Avec une exception pour l'année 2010 qui est celle, comme nous l'avons déjà évoqué, du dossier thématique sur la chronochorématique urbaine, qui explique le pic observé cette année-là des trois catégories Modélisation-Chorème, Urbain et Temps. 
13 Le potentiel heuristique des mots-clés pour embrasser un corpus aussi large et en faire ressortir les axes et évolutions significatives s'avère donc effectif. Ces analyses statistiques, qui passent par une étape de recodage et de classification manuelle toujours discutable, permettent d'incarner l'histoire de la revue. L'éparpillement des mots-clés (on rappelle ici que $40 \%$ des mots-clés, cités une seule fois, n'ont pas été traités) et la réduction en 10 classes qui a été opérée limite, cependant, la finesse de l'analyse. Une exploration des résumés peut alors être envisagée pour évaluer sa complémentarité avec cette première approche.

\section{Exploration des résumés}

14 L'analyse du contenu des résumés apporte davantage de matière à notre observation de l'évolution des thématiques, avec plus de 56000 mots disponibles sur la période, soit environ 59 mots par résumé en moyenne. Cette moyenne peut surprendre, mais, depuis les débuts, les résumés sont traduits en trois langues et pour des raisons de mise en page, dans la version papier, ils étaient très courts (deux à trois phrases). Aujourd'hui la revue demande 600 signes maximum, ce qui laisse aux auteurs un peu plus de marge de manœuvre. Ces ensembles correspondent à 5639 formes actives différentes, c'est-àdire mots expressifs (hors mots-outils), dont 1915 sont cités au moins trois fois dans le corpus.

15 La figure 5 est un nuage de mots comparatif des résumés. Chaque mot est associé à une période par un test du khi-2. La taille des mots est fonction de leur spécificité par rapport à leur groupe d'appartenance. Certains mots très cités peuvent ne pas apparaître, car ils ne sont pas associés significativement à une période donnée. On constate ici aussi une évolution des mots qui est cohérente avec ce qu'on observe pour les mots-clés, à savoir, une concentration sur la première période des mots «cartographie ", «carte» ... Viennent ensuite les «SIG», les «systèmes d'information", puis plus on avance, plus on note une diversité des mots et des nouveaux modes de représentation graphique et cartographique. On note sur la période 2012-2017, par exemple, les mots « roman », « récit », ... 
Figure 5. Nuage de mots comparatif des mots des résumés divisés selon six périodes de temps

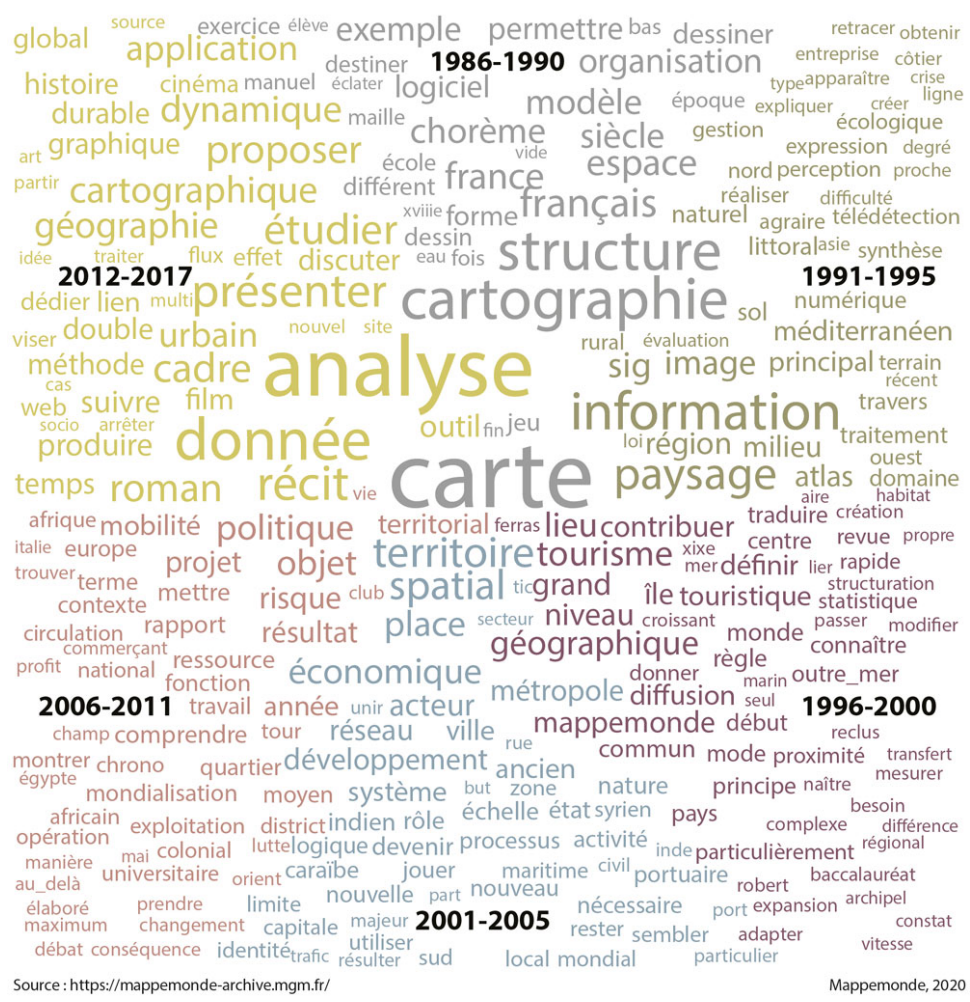

Pour aller au-delà de ce nuage de mots finalement assez complexe, même en le présentant par période, une classification par analyse lexicale a été menée, en utilisant la méthode Reinert disponible dans le logiciel libre Iramuteq ${ }^{6}$. L'analyse produit des regroupements des formes actives en classes selon leur co-présence dans les résumés des articles, sous la forme d'un dendrogramme (figure 6). Sans vouloir entrer ici dans une analyse fine qui serait hors de propos, on peut dégager quelques idées.

Figure 6. Classification par analyse lexicale des résumés (1986-2017)

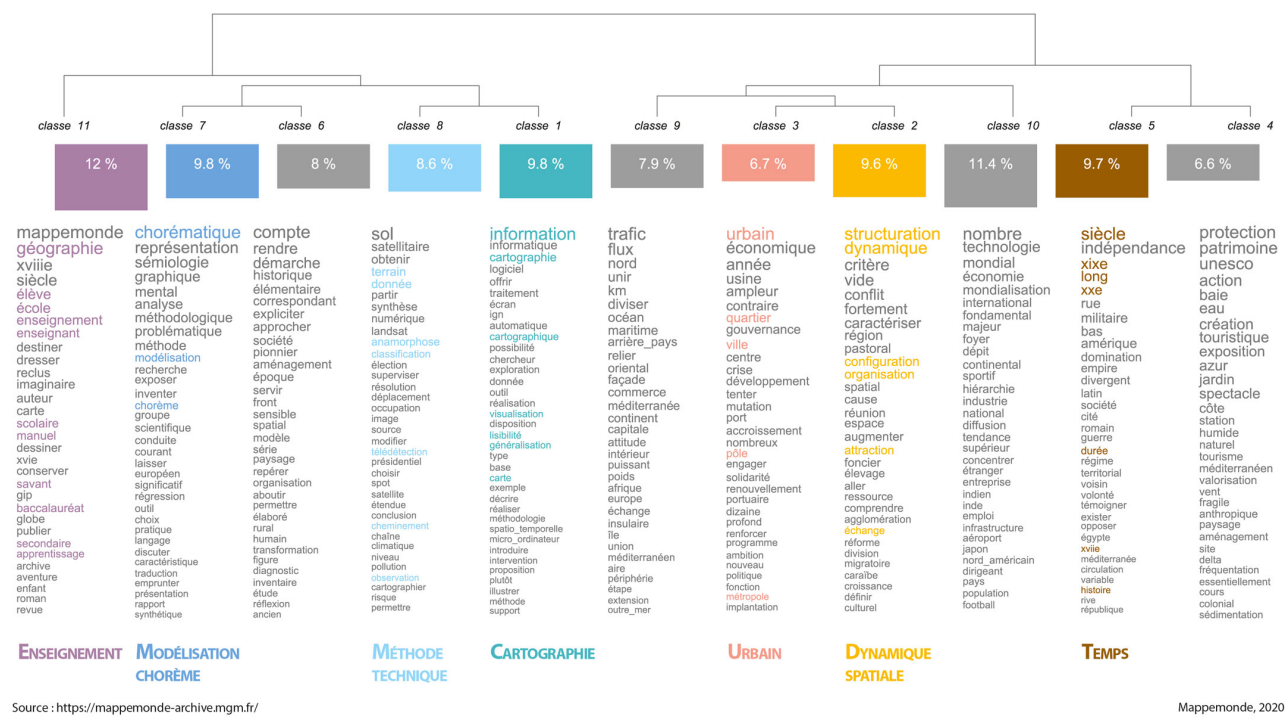

Les catégories ayant le plus de poids (de $9 \%$ à $12 \%$ chacune) correspondent bien aux thèmes préférentiels de la revue tels qu'on se les représente: la géographie, 
l'enseignement, l'étude des dynamiques spatiales, les technologies d'analyse et de représentation et une approche thématique. De plus, les mots actifs dans ces classes permettent de mieux caractériser ces groupes. Ainsi, l'enseignement est associé aux programmes scolaires et, au-delà, au dessin, à l'imaginaire, à la littérature. De même, la modélisation chorématique est associée à la sémiologie, à la recherche, mais aussi à la discussion et à l'exposition. La cartographie est très directement reliée aux logiciels et à l'informatique. Enfin, la thématique de l'histoire est aussi très présente $(9,7 \%)$, avec des périodes et des sujets-clés (XIX ${ }^{\mathrm{e}}$ et $\mathrm{XX}^{\mathrm{e}}$ siècles, les empires, l'histoire militaire...). Une catégorie intermédiaire, avec $8 \%$ des résumés (classe 6 ), semble se centrer autour de l'idée de la communication, de l'explicitation, mais elle est peut-être composée de mots de liaison souvent utilisés dans les résumés pour annoncer l'objectif des articles. D'autres grands thèmes viennent ensuite comme l'approche par les flux et les transports $(7,9 \%)$, l'urbain et l'économie $(6,7 \%)$ ou la protection du patrimoine $(6,6 \%)$.

D'autres analyses seraient intéressantes à mener sur ce corpus, notamment pour évaluer la pertinence des mots-clés associés aux résumés, creuser la signification de certaines formes actives ambiguës ou étonnantes («mental » associé à la chorématique, «terrain » dans les méthodes et techniques...), comparer les poids des pays, continents ou des aires culturelles citées...

\section{Discussion}

La revue Mappemonde est, en 2019, indexée dans différentes bases et moteurs de recherche comme le Directory of Open Access Journal ${ }^{7}$, le JournalBase du CNRS ${ }^{8}$ ou encore l'European reference index for the humanities and social sciences ${ }^{9}$. Cependant, au-delà de l'important travail d'intégration dans ces bases de données nationales ou mondiales, les mots-clés et les résumés occupent un rôle central pour la visibilité des publications scientifiques indexées (Pinto, Lancaster, 1999). Par ailleurs, avec le développement de la folksonomie ${ }^{10}$, l'indexation des documents numériques par l'usager lui-même via l'utilisation des mots-clés (les tags dans la novlangue du web 2.0) se généralise (Le Deuff, 2006). L'analyse exploratoire de ces bases d'indexation - institutionnelles ou collaboratives - crée donc un intérêt croissant à proposer une approche qualitative sur le contenu même de ces corpus, au-delà d'un travail tourné vers l'évaluation et la production d'indicateurs aboutissant à produire des métriques souvent contestables (Gingras, 2014).

20 La tentative proposée ici sur 30 ans d'articles de la revue Mappemonde vise à montrer l'intérêt tout comme les limites de telles approches. Les impressions des lecteurs familiers de la revue sur son orientation et ses contenus se trouvent confortées, mais des résultats plus détaillés ou originaux ressortent aussi des analyses, notamment l'évolution dans le temps de certaines thématiques, avec des «vagues» ou des évolutions cycliques assez bien datées, qui pourront certainement intéresser l'épistémologie des disciplines. "Vagues » et évolutions sont ici illustrées par un bump chart (figure 7) réalisé avec le logiciel Cortext ${ }^{11}$. Il s'agit d'une représentation de l'évolution, au fil des années, de l'occurrence des mots les plus utilisés dans les résumés. 
Figure 7. Bump Chart des mots des résumés utilisés principalement chaque année (1986-2017)

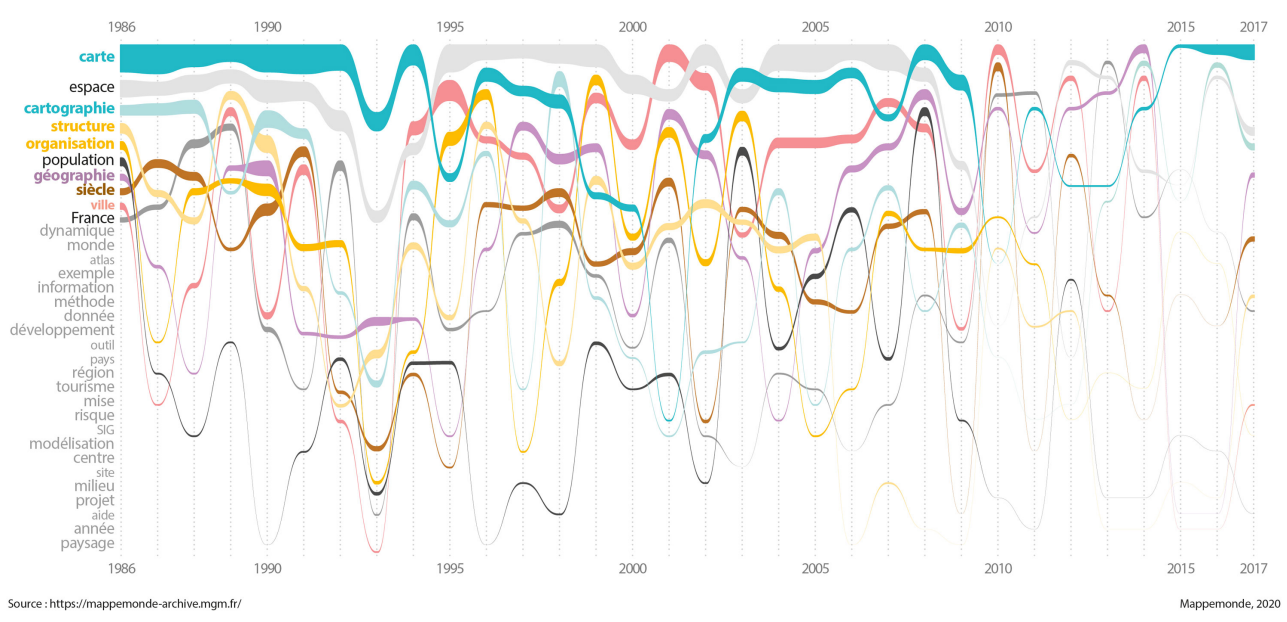

21 Cette visualisation, complexe en première approche, met en valeur les grandes vagues thématiques qui ont fait la revue ainsi que les années atypiques dans l'histoire de Mappemonde. Ainsi, un «creux de la vague » qui correspond à une baisse significative des 10 mots les plus cités sur 30 ans s'observe assez nettement en 1993. Cette année-là, la question du risque apparait et deux numéros thématiques sont publiés («Littoraux » $1 / 93$; « Facette des SIG » 4/93), ce qui explique l'ouverture sur des champs lexicaux un peu différents.

On note encore l'absence de monopole d'un mot particulier voire d'un thème qui conserverait son importance tout au long des 30 ans de Mappemonde. Ce dernier constat illustre bien la diversité de Mappemonde et son ouverture à d'autres champs et modes de représentations, tendance qui s'accentue au fil du temps.

Toutefois, il s'agit là d'une première analyse, qui s'est fondée sur l'utilisation de méthodes textuelles sur lesquelles il est nécessaire de prendre du recul et qu'il conviendrait d'enrichir en proposant des validations supplémentaires. Le fait que les mots-clés n'aient pas été tirés d'une nomenclature, mais laissés au libre choix des auteurs est source de diversité, mais aussi de possibles confusions de sens. Quant aux textes des résumés, ils offrent plus de matière, mais ils emploient souvent des termes descriptifs des objectifs de l'article qu'ils résument qui viennent perturber l'analyse sémantique. Il serait par conséquent heureux d'élargir le corpus, avec, par exemple, la prise en compte des titres des articles, en général assez précis. Avancer en détaillant l'analyse permettrait notamment de mieux cerner ce qui relève de la politique éditoriale de la revue, à différencier, ou pas, de l'évolution de la géographie dans son ensemble ou de manière plus englobante, des sciences du territoire.

En articulant ces analyses automatisées avec les travaux cartographiques des étudiants de Toulouse et Paris 7 (qui seront publiés dans un prochain numéro) et avec l'étude des figures parues depuis 1986 (https://journals.openedition.org/mappemonde/2451), qui abordent la géographie de ce corpus (quels sont les terrains? À quelles échelles privilégiées mobilise-t-on l'image géographique?), l'évolution du paysage de la recherche sur l'image géographique, vue à travers le prisme de la revue, se dessine progressivement. Nul doute que d'autres grilles d'analyse pourraient venir utilement compléter cette première tentative, c'est pourquoi nous tendons la perche aux collègues motivés, le jeu de données est en libre-accès : à vous de jouer !12 


\section{Remarques méthodologiques supplémentaires}

La collecte des mots-clés et résumés s'est opérée par web scraping, une technique d'extraction du contenu de sites web via un script permettant d'automatiser l'opération. Le logiciel libre $\mathrm{R}$ a été utilisé en association avec son package Rvest. Les articles étant répartis sur trois sites web différents (cf. introduction), trois variantes du même script ont été utilisées puis un travail d'intégration au sein d'une même base de données a été réalisé. En cohérence avec la politique éditoriale de la revue, nous diffusons cette base de données en open access. Tout comme les contributions des auteurs, cette base est placée sous licence Creative Commons BY-NC-SA (https:// creativecommons.org/licenses/by-nc-sa/4.0/deed.fr), c'est-à-dire que le partage et l'adaptation des contenus sont autorisés, dans un contexte non commercial et selon les mêmes conditions.

Elle peut être téléchargée à l'adresse suivante: https://analytics.huma-num.fr/ Gregoire.LeCampion/donnees_mappemonde/

Par ailleurs, les auteurs sont preneurs de toutes nouvelles analyses qui viendraient enrichir ce premier travail exploratoire!

\section{BIBLIOGRAPHIE}

ASTÉ J.-P., DUMOLARD P., LÉONE F., JIN S. (1993). « SIG et risques naturels : le glissement de terrain de Séchilienne ». Mappemonde, $\mathrm{n}^{\circ}$ 4. En ligne : https://www.mgm.fr/PUB/Mappemonde/M493/ ROMANCHE.pdf

BRUNET R. (1986). « Mappemonde, le Monde des Cartes ». Mappemonde, $\mathrm{n}^{\circ}$ 1. En ligne : https:// www.mgm.fr/PUB/Mappemonde/M186/p1.pdf

BRUNET R. (2010). « Comprendre la ville par la chrono-chorématique: un essai prometteur ». Mappemonde, $\mathrm{n}^{\circ}$ 100. En ligne : https://mappemonde-archive.mgm.fr/num28/articles/ conclusion.html

FORTON L. 1986. «Structures et dynamique de l'espace en Espagne et en France ». Mappemonde, $\mathrm{n}^{\circ}$ 1. En ligne : https://www.mgm.fr/PUB/Mappemonde/M186/p2-3.pdf

FOVET-RABOT C. (2015). Choisir les mots-clés de l'article scientifique, en 3 points. Montpellier : CIRAD, 2 p. En ligne : https://doi.org/10.18167/coopist/0018

GINGRAS Y. (2014). Les dérives de l'évaluation de la recherche : du bon usage de la bibliométrie. Paris : Raisons d'agir éditions, $128 \mathrm{p}$.

LE DEUFF O. (2006). «Folksonomies ». Bulletin des bibliothèques de France (BBF), n ${ }^{\circ}$ 4, p. 66-70. En ligne : http://bbf.enssib.fr/consulter/bbf-2006-04-0066-002

PINTO M., LANCASTER F.W. (1999). “Abstracts and abstracting in knowledge discovery”. Library trends, vol. $48, \mathrm{n}^{\circ} 1, \mathrm{p} .234-248$ 


\section{NOTES}

1. https://www.mgm.fr/PUB/Mappemonde/MappeSom.html

2. https://mappemonde-archive.mgm.fr/

3. http://mappemonde.mgm.fr/

4. Nous n'avons pas analysé ici les textes de rubriques, qui ne sont pas munis de mots-clés ou de résumés.

5. https://www.mgm.fr/PUB/Mappemonde/M493/ROMANCHE.pdf

6. http://iramuteq.org/

7. https://doaj.org/toc/1769-7298

8. https://journalbase.cnrs.fr/

9. https://dbh.nsd.uib.no/publiseringskanaler/erihplus/periodical/info.action?id=473400

10. Terme proposé par l'architecte de l'information Thomas Vander Wal résultant de la combinaison des mots taxinomie (règles de classification, taxonomy en anglais) et des usagers (folk).

11. https://www.cortext.net/

12. Ce lien mène vers la page des métadonnées de la table : https://www.nakala.fr/page/data/ 11280/85661324. Pour la télécharger, utilisez le lien sous le titre.

\section{RÉSUMÉS}

À l'occasion des 30 ans de la revue Mappemonde, nous proposons d'explorer son contenu par une analyse des 964 articles parus entre 1986 et 2017. L'approche automatisée retenue est focalisée sur les mots-clés et sur les résumés avec, notamment, la production de représentations visuelles expressives. Si elle permet de dessiner à grands traits l'évolution des travaux de recherche publiés dans cette revue sur l'image géographique et les formes du territoire, elle met également en évidence les limites méthodologiques d'un tel exercice. Les données ont été placées en libre accès pour que quiconque puisse reprendre et améliorer l'étude.

For the journal's $30^{\text {th }}$ birthday, Mappemonde has explored its content through the analysis of 964 articles published between 1986 and 2017. Our automated approach focuses on keywords and abstracts, including the production of expressive visual representations. While outlining the evolution of research published in this journal on geographical images and forms of the territory, we have also underlined the methodological limits of such an approach. Data is now open access to allow everyone to continue and improve the study.

Con motivo del treinta aniversario de la revista Mappemonde proponemos explorar su contenido analizando los 964 artículos publicados entre 1986 y 2017. Para valorar la producción de representaciones visuales expresivas se realiza un estudio a partir de las palabras clave y de sus resúmenes. Visibiliza la evolución de las investigaciones publicadas sobre la imagen geográfica y las formas del territorio; pero también muestra las limitaciones metodológicas de estos trabajos. Las bases de información están diponibles gratuitamente para que se pueda continuar y mejorar esta investigación. 
INDEX

Thèmes : 30 ans de publication dans Mappemonde

Mots-clés : rétrospective, analyse textuelle, publication scientifique, thématiques de recherche Palabras claves : retrospectiva, análisis textual, publicación científica, temas de investigación.

Keywords : retrospective, textual analysis, scientific publication, research themes

\section{AUTEURS}

GRÉGOIRE LE CAMPION

CNRS, UMR Passages, Bordeaux

OLIVIER PISSOAT

CNRS, UMR Passages, Bordeaux

\section{LAURENT JÉGOU}

Université de Toulouse-Jean Jaurès, UMR LISST-Cieu

MATTHIEU NOUCHER

CNRS, UMR Passages, Bordeaux 